\title{
Oncolytic herpes simplex virus and temozolomide synergistically inhibit breast cancer cell tumorigenesis in vitro and in vivo
}

\author{
JINGJING FAN $^{1 *}$, HUA JIANG $^{2 *}$, LIN CHENG $^{2}$, BINLIN MA ${ }^{1}$ and RENBIN LIU ${ }^{2}$ \\ ${ }^{1}$ Department of Breast and Neck Surgery, Xinjiang Medical University Affiliated Tumor Hospital, \\ Urumqi, Xinjiang 830011; ${ }^{2}$ Breast Cancer Center, Department of Breast and Thyroid Surgery, \\ The Third Affiliated Hospital of Sun Yat-sen University, Guangzhou, Guangdong 510630, P.R. China
}

Received October 14, 2019; Accepted September 23, 2020

DOI: $10.3892 / \mathrm{ol} .2020 .12360$

\begin{abstract}
The oncolytic herpes simplex virus (HSV) G47\ can selectively eliminate glioblastoma cells via viral replication and temozolomide (TMZ) has been clinically used to treat glioblastoma. However, the combined effect of G47A and TMZ on cancer cells, particularly on breast cancer cells, remains largely unknown. The objective of the present study was to investigate the role and underlying mechanism of G47 $\Delta$ and TMZ, in combination, in breast cancer cell tumorigenesis. The human breast cancer cell lines SK-BR-3 and MDA-MB-468 were treated with G47 $\triangle$ and TMZ individually or in combination. Cell viability, flow cytometry, reverse transcription quantitative-PCR and western blotting were performed to investigate the synergy between G47 $\Delta$ and $\mathrm{TMZ}$ in regulating breast cancer cell behavior in vitro. The role of G47 $\triangle$ and TMZ in suppressing tumorigenesis in vivo was investigated in a xenograft mouse model. G47 $\Delta$ and TMZ served a synergistic role resulting in decreased breast cancer cell viability, induction of cell cycle arrest, promotion of tumor cell apoptosis and enhancement of DNA damage response in vitro. The combined administration of G47A and TMZ also effectively suppressed breast cancer cell-derived tumor growth in vivo, compared with the administration of G47D or
\end{abstract}

Correspondence to: Dr Renbin Liu, Breast Cancer Center, Department of Breast and Thyroid Surgery, The Third Affiliated Hospital of Sun Yat-sen University, 600 Tianhe Road, Guangzhou, Guangdong 510630, P.R. China

E-mail: 461023789@qq.com

Mr. Binlin Ma, Department of Breast and Neck Surgery, Xinjiang Medical University Affiliated Tumor Hospital, Urumqi, Xinjiang 830011, P.R. China

E-mail: mbldoctor@163.com

*Contributed equally

Abbreviations: TMZ, temozolomide; HSV, herpes simplex virus; MOI, multiplicity of infection; CI, combination index

Key words: breast cancer, oncolytic HSV, TMZ, synergy
TMZ alone. Synergy between G47D and TMZ was at least partially mediated via TMZ-induced acceleration of G47 replication, and such a synergy in breast cancer cells in vitro and in vivo provides novel insight into the future development of a therapeutic strategy against breast cancer.

\section{Introduction}

Breast cancer is the most common life-threatening cancer in women globally, with an annual rate of new cases reaching $126 / 100,000$ women and a death rate of $30 \%$ (1). Although conventional treatment options, including surgery, radiotherapy and chemotherapy, can successfully cure patients or prolong patient survival in the majority of cases, each of these therapies has limitations, such as the inability of surgery to eliminate distant metastasis, the lack of durable response to radiotherapy and drug resistance to chemotherapy (2). Therefore, there is an urgent need to develop new effective therapeutic approaches for breast cancer treatment.

Oncolytic viruses can selectively infect, replicate in, and kill cancer cells via induction of cancer cell lysis and the host immune response in infected cancer cells, which results from cancer antigen exposure in lysed cancer cells (3). Notably, oncolytic viruses do not harm healthy cells. Oncolytic virus-based therapy has been regarded as a potential novel therapeutic strategy for cancer treatment (4). Oncolytic viruses have been genetically engineered to improve both the safety of treatment and selectivity (5). Talimogene laherparepvec, a genetically modified herpes simplex virus (HSV), has been approved by the US Food and Drug Administration for clinical application in advanced melanoma therapy (6). Oncolytic HSV G47D is a third-generation replication-competent HSV-1 vector derived from G207 with the deletion of the infected cell protein 47 (ICP47) gene and both copies of the $\gamma 34.5$ gene (7). G47 $\Delta$ has been used to treat glioblastoma in clinical trials in Japan (7).

Temozolomide (TMZ) is an imidazotetrazine-derived alkylating agent used as a first-line oral drug for the treatment of malignant glioma because it is able to easily pass through the blood-brain barrier due to its low molecular weight and lipophilicity $(8,9)$. In addition, TMZ has been used in clinical trials for the treatment of advanced metastatic melanoma $(10,11)$. TMZ kills cancer cells via induction of DNA alkylation and methylation damage in cancer cells (12). 
However, certain types of cancer cells are able to repair TMZ-induced DNA damage, leading to resistance to TMZ, while the genetically modified oncolytic HSV has a decreased replication efficacy in cancer cells compared with naturally occurring HSV, which both decreased the anti-cancer efficacy of such therapies $(13,14)$. Since the therapeutic targets of TMZ and HSV are different, a strategy using a combination of TMZ and HSV may notably enhance the efficacy of cancer therapy via a complementary mechanism.

The present study investigated the combined role of TMZ and $\mathrm{G} 47 \Delta$ in regulating breast cancer cell behavior in vitro and in vivo, and a preliminary mechanism was also suggested. The results of the present study may provide valuable insight into the development of novel therapeutic approaches to treat breast cancer.

\section{Materials and methods}

Cell lines and culture. The human breast cancer cell lines SK-BR-3 and MDA-MB-468, as well as the African green monkey kidney epithelial cell line Vero, were gifts from Dr. Musheng Zeng (Sun Yat-sen University Cancer Center, Guangzhou, China). These cells were grown in Dulbecco's modified Eagle's medium (DMEM; Gibco; Thermo Fisher Scientific, Inc.) supplemented with $10 \%$ inactivated fetal calf serum (FCS; Gibco; Thermo Fisher Scientific, Inc.), 100 IU/ml penicillin, $100 \mu \mathrm{g} / \mathrm{ml}$ streptomycin and $2 \mathrm{mM} \mathrm{L-glutamine.}$ All cells were maintained in a humidified incubator with $5 \%$ $\mathrm{CO}_{2}$ at $37^{\circ} \mathrm{C}$.

Amplification of G47A. G47A was a gift from Dr. Samuel D. Rabkin (Harvard Medical School, Boston, MA, USA) and diluted in $1 \%$ inactivated FCS-containing PBS to infect Vero cells at a multiplicity of infection (MOI) of 0.03 , followed by incubation under standard conditions $\left(5 \% \mathrm{CO}_{2}\right.$ and $\left.37^{\circ} \mathrm{C}\right)$ for 90 min. Viral inoculums were then removed and replaced with $3 \%$ inactivated FCS-containing DMEM, followed by incubation in $5 \% \mathrm{CO}_{2}$ for $48-72 \mathrm{~h}$ at $34.5^{\circ} \mathrm{C}$. Infected cells were collected when $>90 \%$ of the cells appeared round and refractile under a light microscope (magnification, x400) after calculation of 200 cells with two or more fused nuclei vs. the total nuclei (fused and un-fused nuclei) by two investigators and then resuspended in the virus buffer $(20 \mathrm{mM}$ Tris and $150 \mathrm{mM} \mathrm{NaCl}$; $\mathrm{pH}$ 7.5). The resuspension solution was subjected to three rapid freeze-thaw cycles for cell lysis and virus release, followed by centrifugation at $500 \mathrm{x} \mathrm{g}$ and $4^{\circ} \mathrm{C}$ for $10 \mathrm{~min}$. Next, the virus-containing supernatant was collected and stored in multiple aliquots at $-80^{\circ} \mathrm{C}$ until use. The viral titer was determined via a plaque assay according to a previous studies $(15,16)$.

Cell viability assay. SK-BR-3 and MDA-MB-468 cells were seeded into 6 -well plates at a density of $3 \times 10^{5}$ cells/well and $37^{\circ} \mathrm{C}$ incubated for $24 \mathrm{~h}$. The cells were then treated with $2 \mu \mathrm{l}$ DMSO (mock), G47D (MOI, 0.01) and/or TMZ (6 mM for SK-BR-3 and $60 \mathrm{mM}$ for MDA-MB-468; Sigma-Aldrich; Merck KGaA) and cultured in $2 \mathrm{ml}$ DMEM containing $1 \%$ FCS at $37^{\circ} \mathrm{C}$ for $72 \mathrm{~h}$. Next, $5 \mathrm{mg} / \mathrm{ml}$ MTT solution (Sigma-Aldrich; Merck KGaA) was added to each well, and the cell culture was incubated at $37^{\circ} \mathrm{C}$ for additional $4 \mathrm{~h}$. The supernatant was removed carefully, and DMSO was added to dissolve the blue formazan crystals. The optical density was measured at $490 \mathrm{~nm}$.

Chou-Talalay analysis of drug synergy. Chou-Talalay analysis $(17,18)$ was performed to determine the combination index (CI) via assessment of the cell growth inhibition in G47 $\Delta$ and/or TMZ-treated tumor cells using the following equation: $\mathrm{CI}=(D)_{1} /(D x)_{1}+(D)_{2} /(D x)_{2}$, where $(D x)_{1}$ is the dose of agent 1 (e.g. G47 $\Delta$ ) required to produce $\mathrm{x}$ percentage effect alone and $(D)_{1}$ is the dose of agent 1 required to produce the same $x$ percentage effect in combination with $(D)_{2}$. Similarly, $(D x)_{2}$ is the dose of agent 2 (e.g. TMZ) required to produce $\mathrm{x}$ percentage effect alone and $(D)_{2}$ is the dose required to produce the same effect in combination with $(D)_{1}$. The denominators of the aforementioned CI equation, $(D x)_{1}$ and $(D x)_{2}$, can be determined by $D x=D_{m}\left[f_{\mathrm{a}} /\left(1-f_{\mathrm{a}}\right)\right]^{1 / m}$, where $D_{m}$ is the dose required for a $50 \%$ effect (e.g. $50 \%$ inhibition of cell growth), $f_{a}$ is the fraction affected by $D$ (e.g. 0.5 if cell growth is inhibited by 50\%), and $m$ is the coefficient of sigmoidicity of the dose-effect curve. Different values of CI may be obtained to solve the equation for different values of $f_{a}$ (e.g. different degrees of inhibition of cell growth). $\mathrm{CI}<1$ indicates synergy, $\mathrm{CI}>1$ indicates antagonism and $\mathrm{CI}=1$ indicates an additive effect. EnzFitter software, version 1.22 (Biosoft) was used to determine the CI values.

Flow cytometry. A preliminary experiment was performed using SK-BR3 and MDA-MB468 cells after treated with a single drug for $48 \mathrm{~h}$ to determine the $\mathrm{IC}_{50}$. Subsequently, these drug doses were used to assess the effects on tumor apoptosis. In brief, the cells were seeded into a 6-well plate at a density of $3 \times 10^{5}$ cells/well and cultured at $37^{\circ} \mathrm{C}$ for $24 \mathrm{~h}$. The cells were then treated with $2 \mu \mathrm{l}$ DMSO (control), G47 $\Delta$ (MOI, 0.01), and/or TMZ (6 $\mu \mathrm{M}$ for SK-BR-3 and $60 \mu \mathrm{M}$ for MDA-MB-468) in DMEM containing 1\% FCS for $48 \mathrm{~h}$ or $72 \mathrm{~h}$. For the cell cycle analysis, the cells were cultured for $72 \mathrm{~h}$ and then collected, washed with PBS three times and resuspended, followed by overnight fixation in $70 \%$ ethanol at $4^{\circ} \mathrm{C}$. On the next day, the cells were incubated with RNase $\mathrm{A}$ at $37^{\circ} \mathrm{C}$ for $1 \mathrm{~h}$ and then stained with $1 \mu \mathrm{g} / \mathrm{ml}$ propidium iodide (PI) in the dark at $4^{\circ} \mathrm{C}$ for $30 \mathrm{~min}$. Fluorescence of the stained cells was detected for cell cycle analysis via the flow cytometer FACSCanto II (BD Biosciences). The apoptosis assay was performed using an Annexin V PE/7AAD kit (BD Biosciences) according to the manufacturer's protocol. For apoptosis analysis, the cells were cultured for $48 \mathrm{~h}$ and then collected and incubated with $1 \mu \mathrm{g} / \mathrm{ml}$ FITC-Annexin V and $\mathrm{PI}$ in the dark at the room temperature for $10 \mathrm{~min}$, and the fluorescent signals were analyzed using a FACSCanto II flow cytometer (BD Biosciences).

Reverse transcription-quantitative PCR (RT-qPCR). SK-BR-3 and MDA-MB-468 cells were treated as aforementioned. Total RNA was isolated from cells using RNAiso Plus (Takara Biotechnology Co., Ltd.), according to the manufacturer's instructions. cDNA was synthesized via RT of RNA using PrimeScript RT Enzyme Mix and SuperScript ${ }^{\mathrm{TM}}$ III First-Strand Synthesis SuperMix (both Invitrogen; Thermo Fisher Scientific, Inc.) according to the manufacturer's 
protocol. The qPCR products were amplified in the 7500 Real-Time PCR system (Applied Biosystems, Foster city, CA, USA) using SYBR Premix Ex Taq (Invitrogen). The reaction conditions were: $93^{\circ} \mathrm{C}$ For $2 \mathrm{~min}$, then $93^{\circ} \mathrm{C}$ for $1 \mathrm{~min}, 55^{\circ} \mathrm{C}$ for $2 \mathrm{~min}$, for a total of 40 cycles. The primers used are presented in Table I. The relative RNA levels were determined using the $2^{-\Delta \Delta \mathrm{Cq}}(19)$ method and was normalized to $\beta$-actin.

Western blotting. Cells were harvested and lysed in the ice-cold radioimmunoprecipitation assay buffer containing $50 \mathrm{mM}$ Tris- $\mathrm{HCl}, 150 \mathrm{mM} \mathrm{NaCl}, 1 \%$ Triton $\mathrm{X}-100,0.5 \%$ SDS, $1 \mathrm{mM}$ PMSF, $1 \mathrm{mM} \mathrm{Na}_{3} \mathrm{VO}_{4}$, and $0.1 \% \beta$-mercaptoethanol after centrifugation at the top speed $8,000 \mathrm{x} \mathrm{g}$ for $10 \mathrm{~min}$ at $4^{\circ} \mathrm{C}$. The protein concentration was determined via the bicinchoninic acid assay. Protein samples (20 $\mu \mathrm{g}$ each loading) were loaded and separated by SDS-PAGE in $10 \%$ gels, and were then transferred onto nitrocellulose membranes. The membranes were then blocked in $5 \%$ bovine serum albumin (BSA; Sigma-Aldrich; Merck KGaA) in Tris-based saline $0.05 \%$ Tween 20 (TBS-T) for $1 \mathrm{~h}$ at room temperature, followed by overnight incubation with primary antibodies [histone H2AX (H2AX; 1:1,000; cat. no. ab229914), $\gamma$ H2AX (1:1,000; cat. no. ab243906), ATR (1:1,000; cat. no. ab2905), ATM (1:1,000; cat. no. ab32420), DNA-dependent protein kinase, catalytic subunit (DNA-PKc; 1:1,000; cat. no. ab32566), growth arrest and DNA damage-inducible protein GADD34 (GADD34; 1:500; cat. no. ab126075), or $\beta$-actin (1:1,000; cat. no. ab8227); all from Abcam] at $4^{\circ} \mathrm{C}$. Then, membranes were washed three times with TBST and incubated with horseradish peroxidase-conjugated goad anti-mouse IgG (H\&L) (Cat. \#ab6789, Abcam) or rabbit anti-human IgG (H\&L) (Cat. \#ab6759, Abcam; both at a dilution of 1:10,000) for $1 \mathrm{~h}$ at room temperature. After three washes with TBST, the protein bands were detected using enhanced chemiluminescence reagents (cat no. \#35055; Pierce; Thermo Fisher Scientific, Inc.). The western blot imagines were captured and quantified by using a GBOX XT-16 chemiluminescent imager (Syngene) after 20-min exposure of the membranes.

Animal experiments. All animal procedures were approved by the Institutional Animal Care and Use Committee of The Third Affiliated Hospital of Sun Yat-sen University (approval no. 11400700083061). Female BALB/c nude mice with 4 weeks of age and 14-16 g body weight were housed in clean cages and maintained in specific pathogen-free 'barrier' facility with the controlled temperature at $23^{\circ} \mathrm{C}$, the relative humidity of $40-70 \%$, and a 12-h light/dark cycle with free access to food and water. The mice were acclimated for 7 days before the experiments. A total of 28 mice were randomly divided into four groups (n=7/group): Mock, G47 $\Delta$, TMZ and G47 $\Delta+$ TMZ. Mice were intraperitoneally anesthetized with ketamine-xylazine, followed by subcutaneous inoculation with $1 \times 10^{6}$ SK-BR-3 cells in the right hindlimb. Tumor growth was monitored daily, and tumor size was measured every 4 days using a Vernier caliper. The tumor volume was calculated as a $\mathrm{x} \mathrm{b}^{2} / 2$, where $\mathrm{a}$ is the longest and $\mathrm{b}$ is the shortest tumor diameter. When the longest tumor diameter reached $\sim 5 \mathrm{~mm}, \mathrm{TMZ}$ and/or G47 $\Delta$ was administered to the mice, except for those in the Mock group. For the TMZ group, TMZ was intraperitoneally administered once a week at a dose of $50 \mathrm{mg} / \mathrm{kg}$. For the
Table I. Gene-specific primers for quantitative PCR.

\begin{tabular}{|c|c|}
\hline Gene & Sequence, 5'-3' \\
\hline \multirow[t]{2}{*}{ ATM } & Forward: ACTGGCCTTAGCAAATGC \\
\hline & Reverse: TTGCAGCCTCTGTTCGAT \\
\hline \multirow[t]{2}{*}{ ATR } & Forward: TGTCTGTACTCTTCACGGCATGTT \\
\hline & Reverse: AAGAGGTCCACATGTCCGTGTT \\
\hline \multirow[t]{2}{*}{$\mathrm{H} 2 \mathrm{AX}$} & Forward: CAGTGCTGGAGTACCTCAC \\
\hline & Reverse: CTGGATGTTGGGCAGGAC \\
\hline \multirow[t]{2}{*}{ DNA-PKc } & Forward: CTGTGCAACTTCACTAAGTCCA \\
\hline & Reverse: CAATCTGAGGACGAATTGCCT \\
\hline \multirow[t]{2}{*}{ GADD34 } & Forward: GGAGGAAGAGAATCAAGCCA \\
\hline & Reverse: TGGGGTCGGAGCCTGAAGAT \\
\hline \multirow[t]{2}{*}{ RRM1 } & Forward: TGGCCTTGTACCGATGCTG \\
\hline & Reverse: GCTGCTCTTCCTTTCCTGTGTT \\
\hline \multirow[t]{3}{*}{ RRM2 } & Forward: GCGATTTAGCCAAGAAGTTCA \\
\hline & GAT \\
\hline & Reverse: CCCAGTCTGCCTTCTTCTTGA \\
\hline \multirow[t]{3}{*}{$\beta$-actin } & Forward: TGGCACCCAGCACAATGAA \\
\hline & Reverse: CTAAGTCATAGTCCGCCTAGAA \\
\hline & GCA \\
\hline
\end{tabular}

$\mathrm{H} 2 \mathrm{AX}$, histone H2AX; GADD34, growth arrest and DNA damage-inducible protein GADD34; DNA-PKc, DNA-dependent protein kinase, catalytic subunit; RRM, ribonucleotide reductase catalytic subunit M1; RRM2, ribonucleotide reductase catalytic subunit M2.

G47 $\Delta$ group, G47 $\Delta$ was injected intratumorally once every 3 days for a total of four times at a dose of $1 \times 10^{6} \mathrm{pfu} / \mathrm{mouse}$. In the G47 $\Delta$ plus TMZ group, TMZ and G47 $\Delta$ were administered in combination in the aforementioned manner. The mice were sacrificed 60 days after inoculation or when the longest tumor diameter reached $18 \mathrm{~mm}$ via $\mathrm{CO}_{2}$ and cervical dislocation and all tumor xenografts were taken and analyzed using different assays (see details in the corresponding methods parts).

Statistical analysis. The data were expressed as the mean \pm standard deviation of the triplicated experiments and were statistically analyzed using SPSS software (version 13.0; SPSS, Inc.). Comparisons between two groups were conducted using the Student's t-test and Pearson correlation analysis was used to assess correlation. Comparisons of multiple groups was analyzed using the one-way ANOVA followed by Bonferroni's correction. $\mathrm{P}<0.05$ was considered to indicate a statistically significant difference.

\section{Results}

G47A and TMZ synergistically inhibit breast cancer cell viability in vitro. In order to investigate the combined effect of G47 $\triangle$ and TMZ on breast cancer cells, the individual effects of G47 $\Delta$ and TMZ on the viability of SK-BR-3 and MDA-MB-468 cells were assessed. Treatment with G47 $\Delta$ or TMZ alone inhibited viability of SK-BR-3 and MDA-MB-468 cells in a dose-dependent manner, with median effective doses 


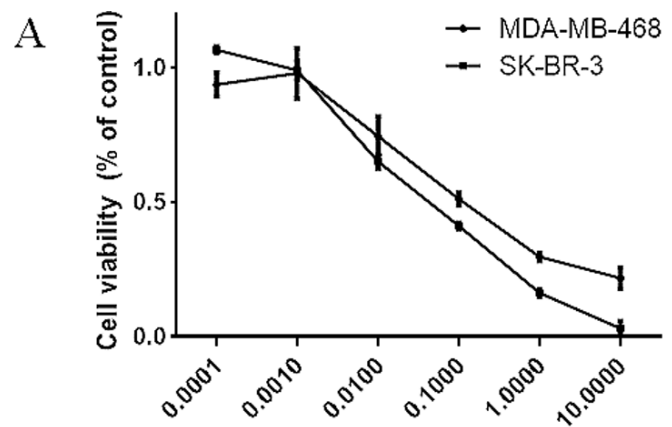

$\mathrm{C}$

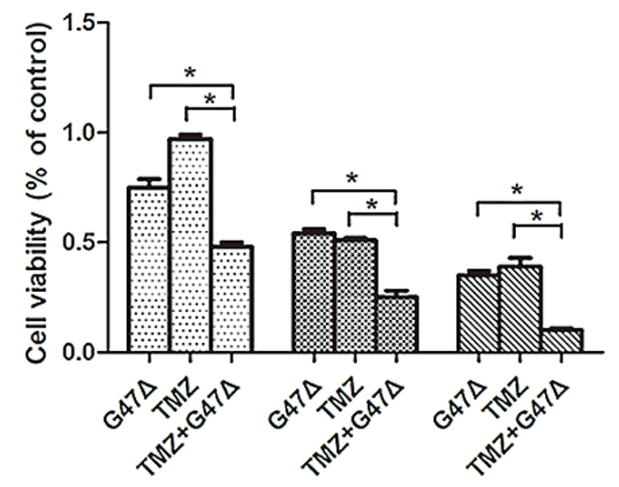

E

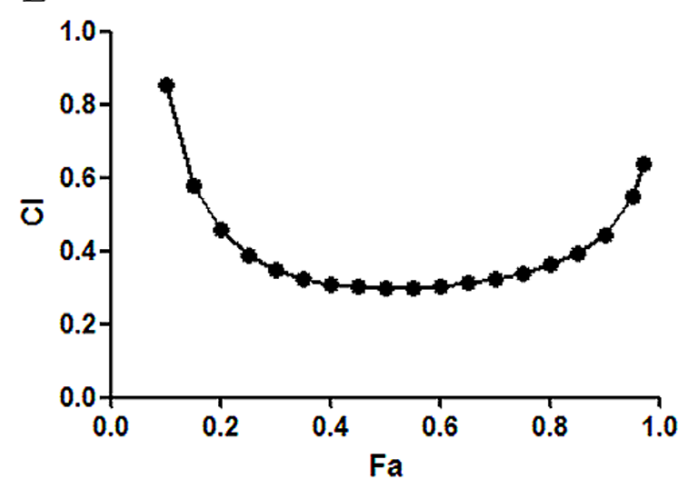

$\mathrm{B}$

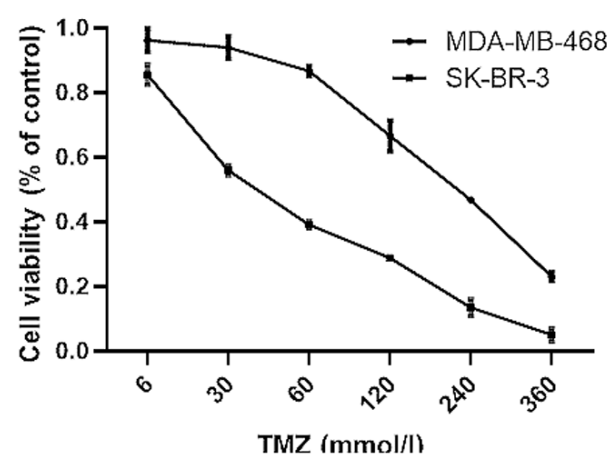

$\mathrm{MOl} / \mu \mathrm{mol} / \mathrm{l} \quad \mathrm{D}$

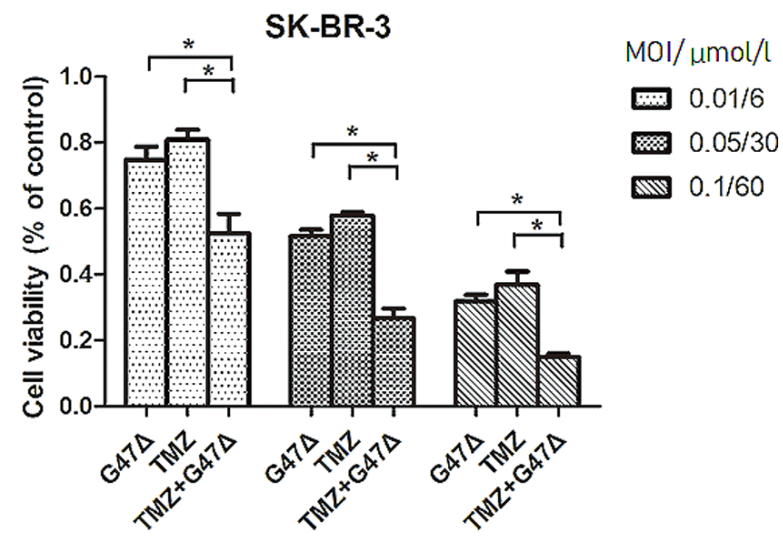

$\mathrm{F}$

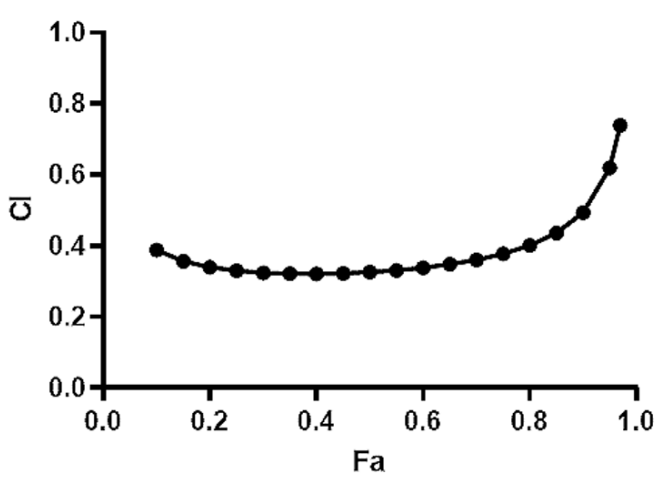

Figure 1. Synergistic inhibitory effect of G47 $\Delta$ and TMZ on breast cancer cell viability. The individual effect of (A) G47 $\Delta$ or (B) TMZ on the proliferation of SK-BR-3 and MDA-MB-468 cells at different doses was assessed via MTT assay. The combined effect of G47 $\triangle$ and TMZ on the viability of (C) MDA-MB-468 and (D) SK-BR-3 cells at different doses was determined via MTT assay. The synergistic effect between G47 $\Delta$ and TMZ on inhibition of (E) MDA-MB-468 and (F) SK-BR-3 cell viability was determined via Chou-Talalay analysis. "P<0.05. TMZ, temozolomide; CI, combination index; MOI mulplicity of infection.

$\left(\mathrm{ED}_{50}\right)$ of 0.09 and $0.20 \mathrm{MOI}$ for $\mathrm{G} 47 \Delta$, and 36.6 and $171.9 \mu \mathrm{M}$ for TMZ in SK-BR-3 and MDA-MB-468 cells, respectively (Fig. 1A and B). G47D and TMZ in combination further suppressed breast cancer cell viability in a dose-dependent manner, compared with G47 $\Delta$ or TMZ alone (Fig. 1C and D), indicating a potential synergy between G47 $\Delta$ and TMZ in inhibiting cell viability. In order to determine whether synergy existed between G47 $\Delta$ and TMZ, the Chou-Talalay analysis was performed for multiple G47 $\Delta / \mathrm{TMZ}$ ratios. CI was 0.39-0.74 (CI, <0.9) in SK-BR-3 cells and 0.64-0.85 (CI, <0.9) in MDA-MB-468 cells (Fig. 1E and F). These results indicated that G47 $\triangle$ and TMZ exhibited a synergistic inhibitory effect on breast cancer cell growth.

G474 and TMZ synergistically induce breast cancer cell cycle arrest in vitro. Cell cycle progression is associated with cell division and growth (20). The combined effect of G47 $\Delta$ and TMZ on inhibition of breast cancer cell cycle progression was investigated. G47 $\Delta$ treatment alone induced SK-BR-3 and MDA-MB-468 cell cycle arrest at the $G_{0} / G_{1}$ phase of the cell cycle $(52.1 \pm 2.1$ and $58.9 \pm 3.6 \%$, respectively), whereas TMZ alone induced SK-BR-3 and MDA-MB-468 cell cycle arrest at the $\mathrm{G}_{2} / \mathrm{M}$ phase $(39.9 \pm 3.7$ and $30.2 \pm 4.1 \%$, respectively) (Fig. 2). By contrast, a combination of G47D and TMZ notably arrested the cell cycle at the $\mathrm{G}_{0} / \mathrm{G}_{1}$ phase in SK-BR-3 cells (71.4 \pm 1.0 vs. $\mathrm{G} 47 \Delta 52.1 \pm 2.1 \% ; \mathrm{P}=0.003)$ and at the $\mathrm{G}_{2} / \mathrm{M}$ phase in MDA-MB-468 cells (41.5 \pm 2.0 vs. TMZ 30.2 $\pm 4.1 \%$; $\mathrm{P}=0.012)$. These data indicated that $\mathrm{G} 47 \Delta$ and $T M Z$ in combination exhibited a more significant suppressive effect on breast cancer cell cycle progression than G47 $\Delta$ or TMZ alone, suggesting a synergy between G47 $\triangle$ and TMZ in the induction of breast cancer cell cycle arrest. 

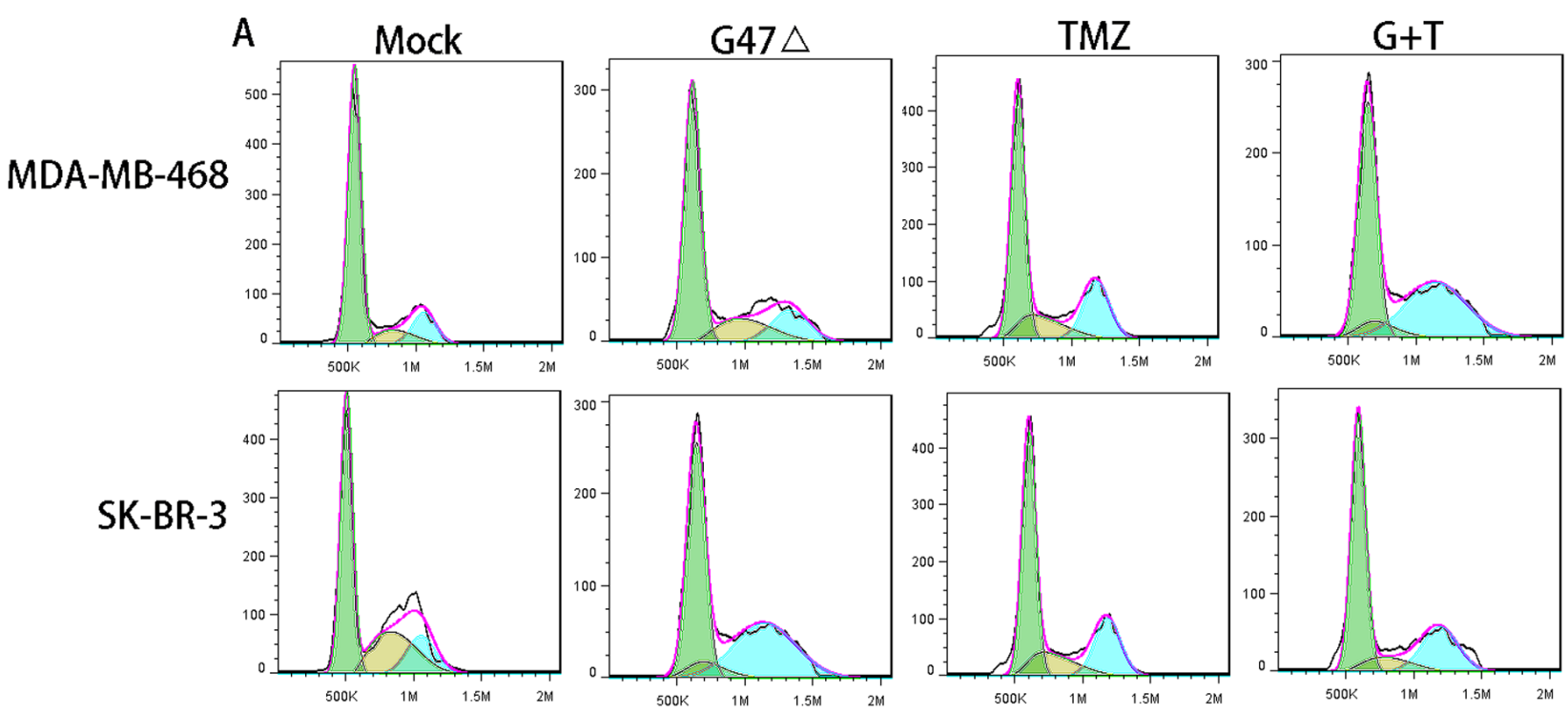

$\mathrm{B}$

MDA-MB-468
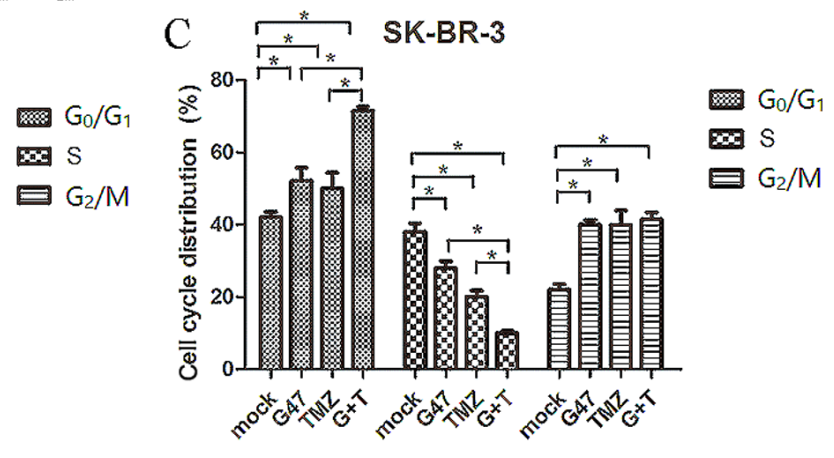

Figure 2. Synergistic inductive effect of G47 $\triangle$ and TMZ on breast cancer cell cycle arrest. (A) Cell cycle was analyzed via flow cytometry. Histograms are presented for the cell cycle analysis of (B) MDA-MB-468 and (C) SK-BR-3 cells following treatment with G47D and TMZ individually or in combination for 72 h. ${ }^{*} \mathrm{P}<0.05$. TMZ, temozolomide; G+T, G47 $\Delta$ + TMZ.

G474 and TMZ synergistically promote breast cancer cell apoptosis in vitro. Cell apoptosis is a key process during tumor development (21). Therefore, it was investigated whether G47 $\triangle$ and TMZ may exhibit a synergistic effect on breast cancer cell apoptosis. G47 $\triangle$ and TMZ individually markedly induced SK-BR-3 cell apoptosis, compared with the mock control group $(37.10 \pm 2.30$ and $25.50 \pm 2.60$ vs. $1.70 \pm 0.26 \%$, respectively) (Fig. 3). Combined G47 $\triangle$ and TMZ significantly promoted SK-BR-3 cell apoptosis $(59.60 \pm 2.25$ vs. $37.10 \pm 2.30$ and $25.50 \pm 2.60 \%$; both $\mathrm{P}<0.05)$. Similarly, a combination of G47 $\triangle$ and TMZ further enhanced MDA-MB-468 cell apoptosis $(40.2 \pm 1.1$ vs. $25.1 \pm 4.4$ and $24.4 \pm 5.1 \%$, respectively; both $\mathrm{P}<0.05$ vs. control group). Collectively, these data suggested that G47D and TMZ synergistically decreased tumor cell proliferation via induction of breast cancer cell apoptosis.

G47A and TMZ synergistically regulate the expression levels of DNA damage-associated genes in breast cancer cells. Cell cycle arrest and apoptosis are triggered by DNA damage in cells (22). Therefore, it was determined whether G47 $\triangle$ and TMZ serve a synergistic role in induction of DNA damage in breast cancer cells. The results showed that G47A or TMZ alone induced expression of $\gamma \mathrm{H} 2 \mathrm{AX}$ protein but not the H2AX mRNA level; $\gamma \mathrm{H} 2 \mathrm{AX}$ is a sensitive molecular marker of DNA double-strand breaks (23), in SK-BR-3 cells (Figs. 4 and 5). G47D and TMZ in combination further augmented the individual effect of G47 $\Delta$ or
TMZ on the mRNA and protein expression levels of H2AX and $\gamma \mathrm{H} 2 \mathrm{AX}$, respectively. In addition, G47D and TMZ in combination notably promoted G47A- or TMZ-induced expression levels of GADD34, TM, DNA-PKc, RRM1, RRM2, and ATR, which are key DNA damage response genes (24). Similar results were also observed in MDA-MB-468 cells (Figs. 4 and 5). These findings indicated that G47 $\Delta$ and TMZ synergistically upregulated the expression levels of DNA damage-associated genes, and may thus induce DNA damage and trigger the DNA damage response, which in turn leads to breast cancer cell cycle arrest and apoptosis (Fig. 3).

G47A and TMZ synergistically suppress breast cancer cell-derived tumor growth in vivo. In order to further investigate the combined role of G47 $\triangle$ and TMZ in breast cancer cell tumorigenesis in vivo, a breast cancer xenograft model was established by inoculating SK-BR-3 cells into nude mice, which were then treated with G47 $\triangle$ and TMZ individually or in combination. As shown in Fig. 6, G47 $\Delta$ or TMZ alone significantly decreased the size of SK-BR-3 cell-derived tumor xenografts in a time-dependent manner, compared with the mock group [519.0 $\pm 133.3(\mathrm{n}=6)$ and $591.3 \pm 41.8(\mathrm{n}=7)$ vs. $1,402.3 \pm 375.3 \mathrm{~mm}^{3}$ $(n=6)$ at 36 days after inoculation, respectively; both $\mathrm{P}<0.05]$. A combination of G47 $\triangle$ and TMZ further enhanced the inhibitory effect of individual G47 $\Delta$ or TMZ on tumor growth [125.0 \pm 7.6 $(n=7)$ vs. $519.0 \pm 133.3(n=6)$ and $591.3 \pm 41.8 \mathrm{~mm}^{3}(n=7)$ at 36 days 
A MDA-MB-468
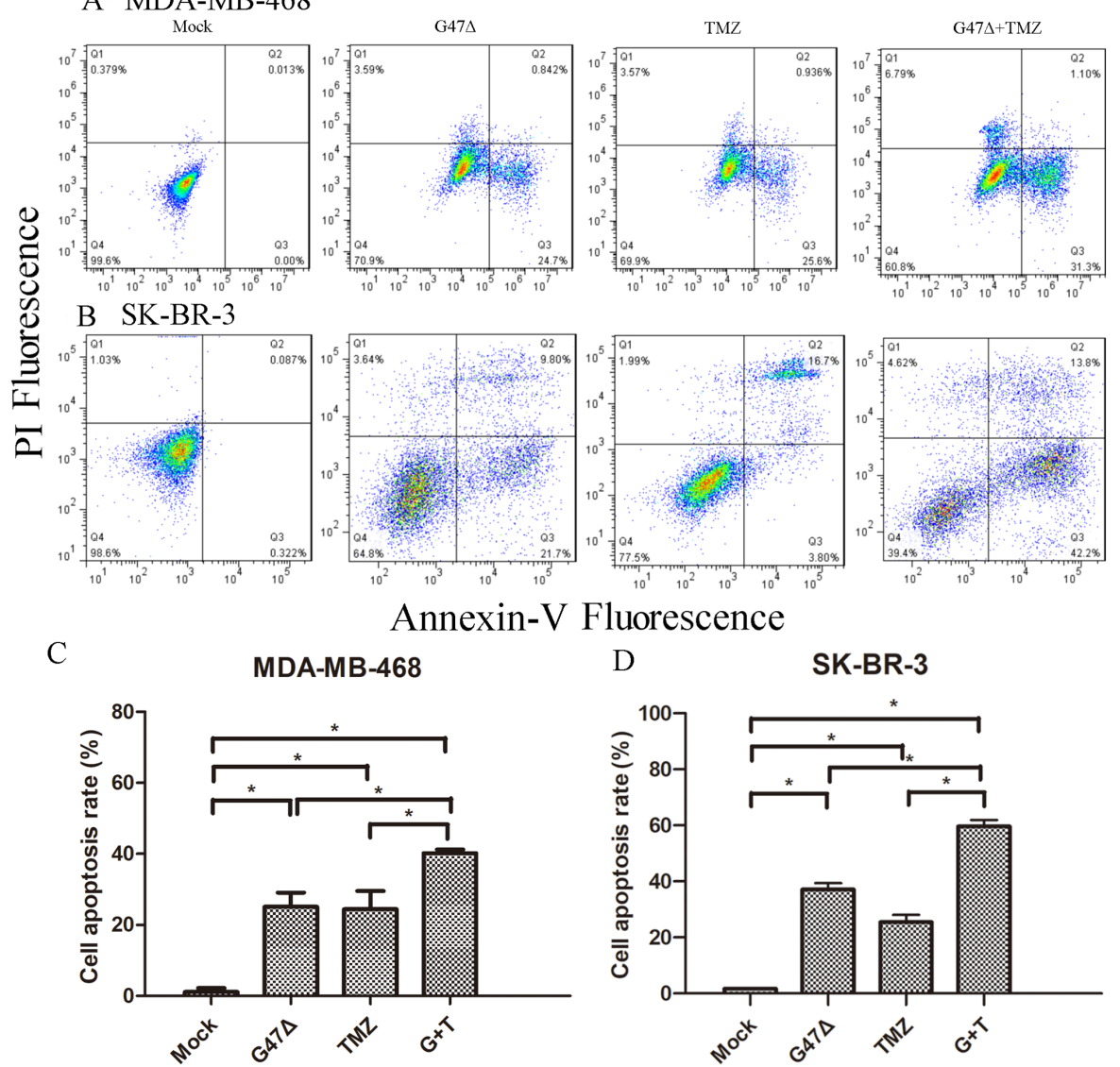

uorescence

$\mathrm{D}$

SK-BR-3

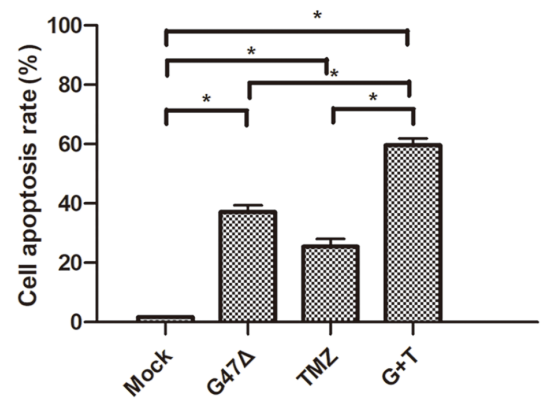

Figure 3. Synergistic inductive effect of G47D and TMZ on breast cancer cell apoptosis. Cell apoptosis was analyzed in (A) MDA-MB-468 and (B) SK-BR-3 cells treated with G47 $\mathrm{A}$ and TMZ individually or in combination for $48 \mathrm{~h}$ via flow cytometry. Apoptotic rates of (C) MDA-MB-468 and (D) SK-BR-3 cells treated with G47 $\Delta$ and TMZ individually or in combination were quantified. ${ }^{*} \mathrm{P}<0.05$. TMZ, temozolomide; PI, propidium iodide; G+T, G47 + TMZ.
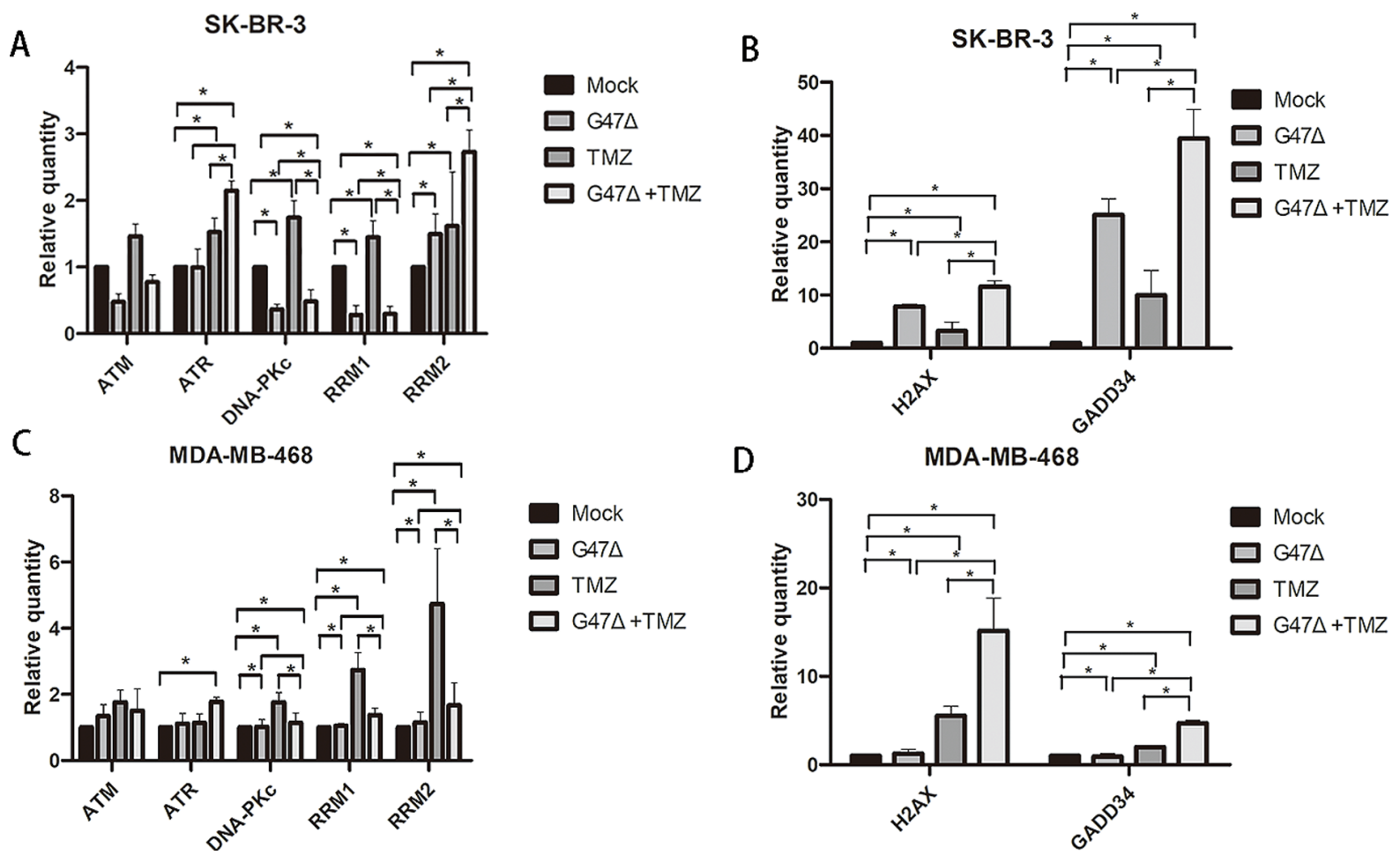

Figure 4. Synergistic inductive effect of G47D and TMZ on DNA damage in breast cancer cells. Reverse transcription-quantitative PCR was performed to assess the expression levels of DNA damage-associated genes in (A and B) SK-BR-3 and (C and D) MDA-MB-468 cells following treatment with G47D and TMZ individually or in combination for $48 \mathrm{~h}$. ${ }^{*} \mathrm{P}<0.05$. TMZ, temozolomide; H2AX, histone H2AX; GADD34, growth arrest and DNA damage-inducible protein GADD34; DNA-PKc, DNA-dependent protein kinase, catalytic subunit. 


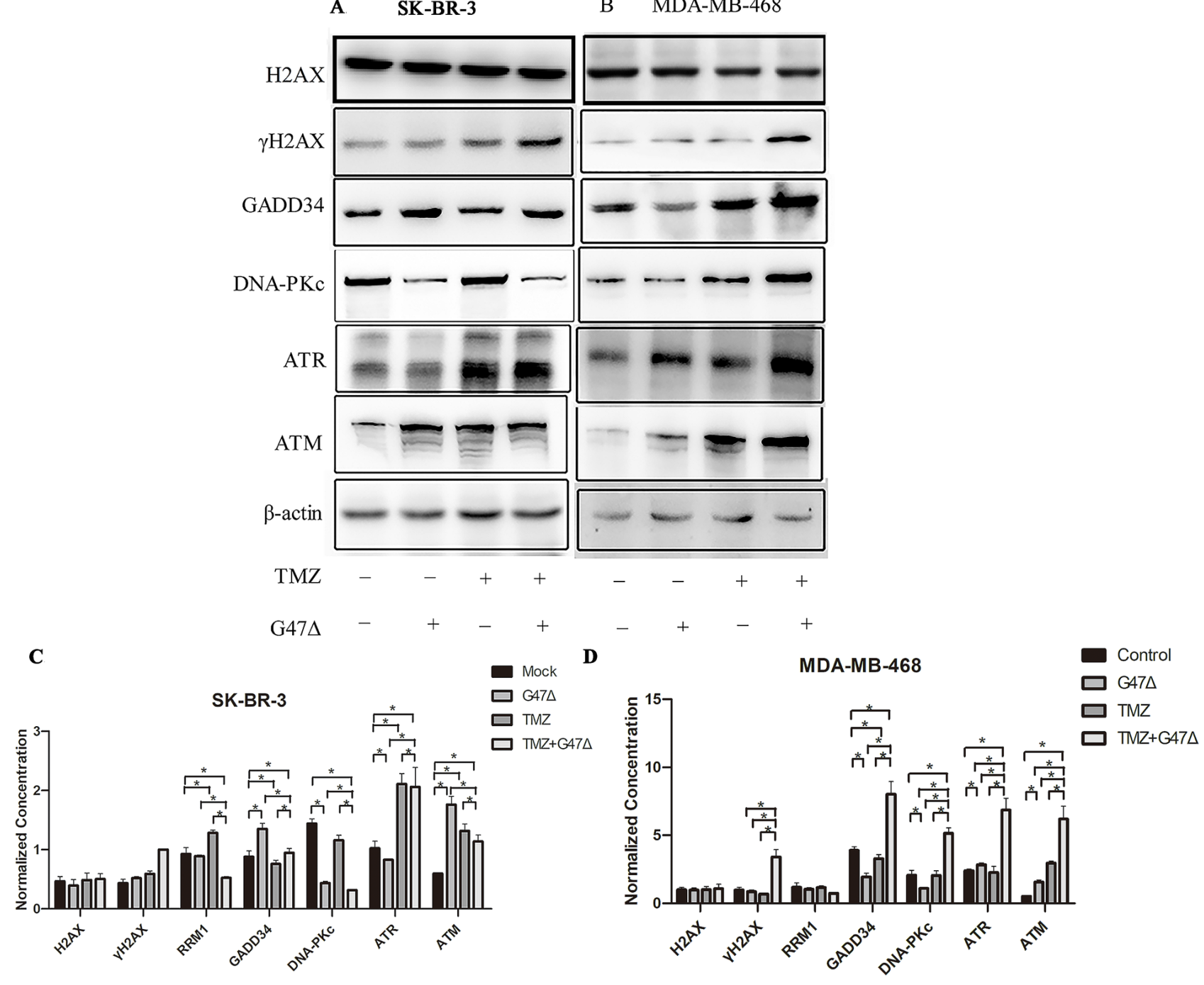

Figure 5. Synergistic inductive effect of G47 $\triangle$ and TMZ on DNA damage in breast cancer cells. Western blotting was used to detect expression levels of the indicated DNA damage-associated proteins in (A) SK-BR-3 and (B) MDA-MB-468 cells following treatment with G47D and TMZ individually or in combination for 48 h. Semi-quantification of (C) SK-BR-3 and (D) MDA-MB-468 western blotting results. "P<0.05. TMZ, temozolomide; H2AX, histone H2AX; GADD34, growth arrest and DNA damage-inducible protein GADD34; DNA-PKc, DNA-dependent protein kinase, catalytic subunit.

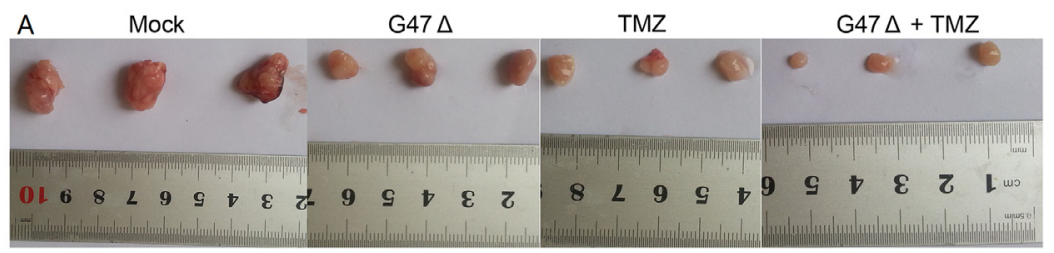

B

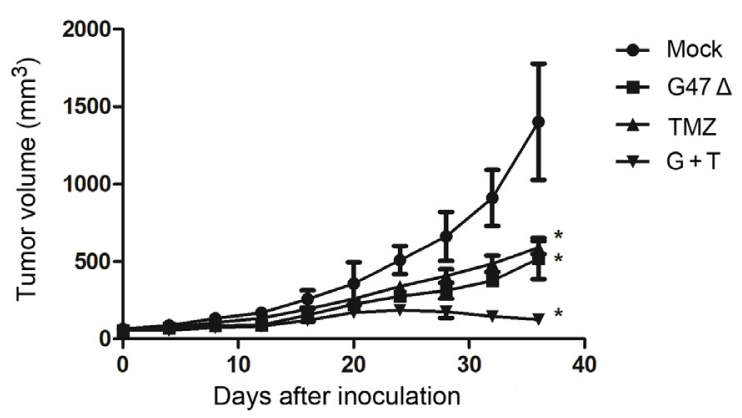

Figure 6. Synergistic inhibitory effect of G47 $\mathrm{and}$ TMZ on breast cancer cell tumorigenesis in vivo. Female nude mice (age, 4 weeks) were inoculated with SK-BR-3 cells, followed by treatment with G47 $\Delta$ and TMZ individually or in combination when tumor xenografts reached $5 \mathrm{~mm}$ in the longest diameter. The tumor size was measured every 4 days after tumor cell inoculation. (A) Tumor xenografts. (B) Tumor volume. "P<0.05 vs. mock control. TMZ, temozolomide; $\mathrm{G}+\mathrm{T}, \mathrm{G} 47 \Delta$ and TMZ. 
A

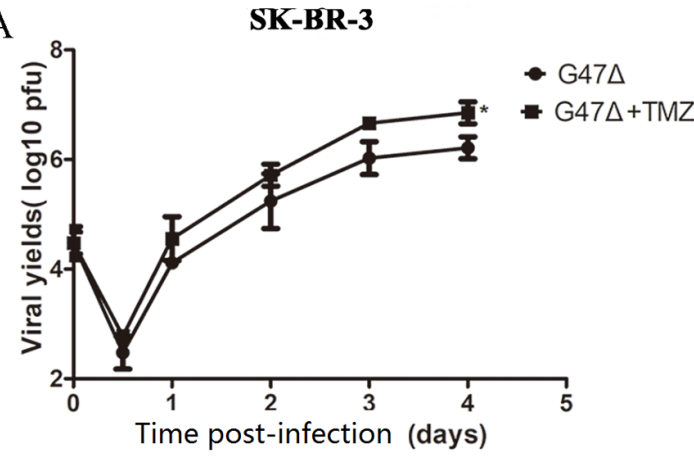

$\mathrm{B}$

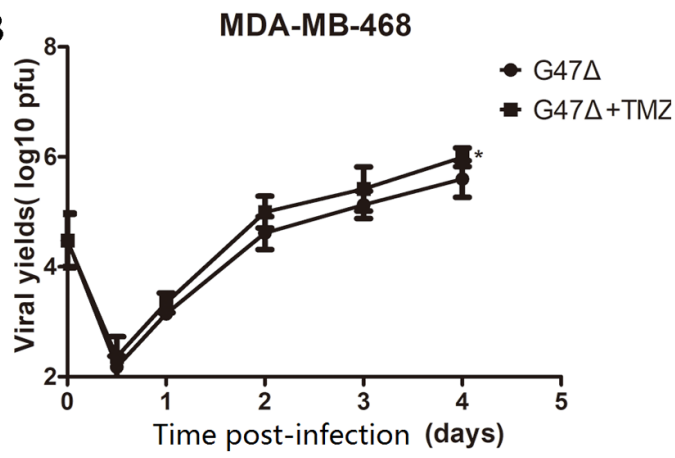

Figure 7. TMZ promotion of G47 $\Delta$ replication. (A) SK-BR-3 and (B) MDA-MB-468 cells were cultured for $24 \mathrm{~h}$ and then treated with G47 $\Delta$ alone in combination with TMZ for the indicated periods of time. G47A was then collected to determine the viral titer via plaque assay. ${ }^{*}<<0.05$ using Student's t-test. TMZ, temozolomide.

after inoculation, respectively; both $\mathrm{P}<0.05$ vs. control group]. These data indicated that combined treatment with G47 $\Delta$ and $\mathrm{TMZ}$ decreased breast cancer cell-derived tumor growth more effectively than treatment with G47 $\Delta$ or TMZ alone, suggesting a synergy between $\mathrm{G} 47 \Delta$ and TMZ in suppressing breast cancer cell tumorigenesis in vivo.

$T M Z$ accelerates $G 474$ replication in vitro. The mechanism underlying the synergistic inhibitory effect of G47D and TMZ on breast cancer cell tumorigenesis was further investigated. The yield of G47 $\Delta$ increased in a time-dependent manner in SK-BR-3 and MDA-MB-468 cells treated with G47D and TMZ together, compared with that in cells treated with G47 $\Delta$ alone (Fig. 7). These findings indicated that a synergy between G47 $\Delta$ and TMZ in inhibiting breast cancer cell tumorigenesis may be at least partially due to acceleration of G47A replication by TMZ.

\section{Discussion}

Genetically modified replication-competent oncolytic HVS strains have been used as oncolytic virus therapy in which cancer cells are killed via a direct oncolytic effect of the virus and induction of host immunity (25). However, due to the highly attenuated replication ability of these genetically altered viruses, including HSV G47 $\Delta$, oncolytic virus therapy is commonly used in combination with chemotherapy or radiotherapy to improve the efficiency of cancer treatment.

In the present study, the combination of G47 $\Delta$ and TMZ induced stronger cytotoxicity than G47 $\Delta$ or $\mathrm{TMZ}$ alone in breast cancer cells. This synergy is likely due to the distinct mechanisms of G47 $\Delta$ and TMZ in killing breast cancer cells. For example, TMZ induces cancer cell DNA damage/repair, as evidenced by TMZ-induced upregulation of the DNA damage response genes ATR and GADD34. By contrast, G47D replicates in and lyses cancer cells. A previous study demonstrated that TMZ-induced DNA repair notably enhanced HSV-mediated oncolysis in primary brain tumor cells via promotion of HSV replication (26). Therefore, TMZ treatment may induce the sensitivity of breast cancer cells to G47 $\Delta$ infection, resulting in accelerated G47 $\Delta$ replication and augmented cancer cell lysis. The present study confirmed that $\mathrm{TMZ}$ may promote $\mathrm{G} 47 \Delta$ replication in breast cancer cells in vitro.

The present study also demonstrated that G47D and TMZ in combination synergistically induced breast cancer cell apoptosis. A previous study demonstrated that HSV can induce robust apoptosis of TMZ-resistant glioma cells both in vitro and in vivo, indicating synergy between G47 $\triangle$ and TMZ (27). DNA damage promotes cell apoptosis if such DNA damage is not repaired $(22,28,29)$. In TMZ-resistant breast cancer, repairing TMZ-induced DNA damage promotes G47 $\Delta$ replication in breast cancer cells. These cells are lysed, triggering the host immune response and resulting in cytokine-induced cancer cell apoptosis (30). In addition, the synergistic role of G47 $\Delta$ and TMZ in DNA damage may interfere with DNA replication in breast cancer cells, further affecting cancer cell division. The present study demonstrated that G47D and TMZ exhibited a synergistic effect on induction of breast cancer cell cycle arrest. The cell cycle was arrested at different phases in SK-BR-3 and MDA-MB-468 cells, which is likely due to the different genetic background between these two cell lines (31).

The synergy between G47 $\Delta$ and TMZ in regulation of breast cancer cell behavior was further verified in a nude mouse xenograft model. The in vivo synergy in inhibition of breast cancer cell-derived tumor xenograft growth may be due to the effects of treatments on breast cancer cell viability, apoptosis, cycle arrest and DNA damage/repair. The present study may provide valuable information for the potential clinical application of G47 $\Delta$ and TMZ in breast cancer treatment.

However, the present study has certain limitations. For example, although the combined effect of G47D and TMZ on breast cancer cell behaviors (due to acceleration of G47A replication by TMZ) was demonstrated, the underlying mechanism by which TMZ promoted G47D replication was not investigated in detail. Moreover, it was not assessed whether G47 $\Delta$ may serve a role in increasing the sensitivity of breast cancer cells to TMZ. The present study also did not identify the optimal dose combination of G47 $\triangle$ and TMZ to suppress breast cancer cell tumorigenesis in vitro and in vivo. Finally, clinical or preclinical data were not available to assess the therapeutic value of G47 $\Delta$ and TMZ in combination for breast cancer development and progression, although previous studies have demonstrated anti-breast cancer activity in vitro (32-34). Further investigation is required to elucidate these points. In conclusion, the present study demonstrated that the combined administration of G47 $\triangle$ and TMZ effectively suppressed breast cancer cell-derived tumor growth in vivo, compared with the administration of G47 $\Delta$ or TMZ alone. Synergy 
between G47 $\triangle$ and TMZ was at least partially mediated via TMZ-induced acceleration of G47 $\Delta$ replication, and such a synergy in breast cancer cells in vitro and in vivo provides novel insight into the future development of a therapeutic strategy against breast cancer.

\section{Acknowledgements}

Not applicable.

\section{Funding}

The present study was funded in part by a grant from the Xinjiang Medical University Research and Innovation Fund Project (grant no. XYDCX201677).

\section{Availability of data and materials}

The datasets used and/or analyzed during the current study are available from the corresponding author on reasonable request.

\section{Authors' contributions}

JF and RL designed the research. JF performed the main experiments and drafted the paper. HJ participated in the construction of the animal models. LC analyzed the data. BM participated the study design, interpreted the data and prepared the manuscript. All authors read and approved the final manuscript.

\section{Ethics approval and consent to participate}

All animal procedures were approved by the Institutional Animal Care and Use Committee of The Third Affiliated Hospital of Sun Yat-sen University (approval no. 11400700083061 ).

\section{Patient consent for publication}

Not applicable.

\section{Competing interests}

The authors declare that they have no competing interests.

\section{References}

1. Shah R, Rosso K and Nathanson SD: Pathogenesis, prevention, diagnosis and treatment of breast cancer. World J Clin Oncol 5: 283-298, 2014.

2. Tinoco G, Warsch S, Gluck S, Avancha K and Montero AJ Treating breast cancer in the 21st century: Emerging biological therapies. J Cancer 4: 117-132, 2013.

3. Lou E: Oncolytic herpes viruses as a potential mechanism for cancer therapy. Acta Oncol 42: 660-671, 2003.

4. Chiocca EA and Rabkin SD: Oncolytic viruses and their application to cancer immunotherapy. Cancer Immunol Res 2: 295-300, 2014.

5. Burke J, Nieva J, Borad MJ and Breitbach CJ: Oncolytic viruses: Perspectives on clinical development. Curr Opin Virol 13: 55-60, 2015.

6. Rehman H, Silk AW, Kane MP and Kaufman HL: Into the clinic: Talimogene laherparepvec (T-VEC), a first-in-class intratumoral oncolytic viral therapy. J Immunother Cancer 4: 53, 2016.
7. Fukuhara $\mathrm{H}$, Ino $\mathrm{Y}$ and Todo T: Oncolytic virus therapy: A new era of cancer treatment at dawn. Cancer Sci 107: 1373-1379, 2016.

8. Mannas JP, Lightner DD, Defrates SR, Pittman T and Villano JL: Long-term treatment with temozolomide in malignant glioma. J Clin Neurosci 21: 121-123, 2014.

9. Sengupta S, Marrinan J, Frishman C and Sampath P: Impact of temozolomide on immune response during malignant glioma chemotherapy. Clin Dev Immunol 2012: 831090, 2012.

10. Bafaloukos D, Tsoutsos D, Kalofonos H, Chalkidou S, Panagiotou P, Linardou E, Briassoulis E, Efstathiou E, Polyzos A, Fountzilas G, et al: Temozolomide and cisplatin versus temozolomide in patients with advanced melanoma: A randomized phase II study of the hellenic cooperative oncology group. Ann Oncol 16: 950-957, 2005.

11. Dronca RS, Allred JB, Perez DG, Nevala WK, Lieser EA, Thompson M, Maples WJ, Creagan ET, Pockaj BA, Kaur JS, et al: Phase II study of temozolomide (TMZ) and everolimus (RAD001) therapy for metastatic melanoma: A north central cancer treatment group study, N0675. Am J Clin Oncol 37: 369-376, 2014.

12. Zhang J, Stevens MF and Bradshaw TD: Temozolomide: Mechanisms of action, repair and resistance. Curr Mol Pharmacol 5: 102-114, 2012.

13. Kondo N, Takahashi A, Mori E, Noda T, Zdzienicka MZ, Thompson LH, Helleday T, Suzuki M, Kinashi Y, Masunaga S, et al: FANCD1/BRCA2 plays predominant role in the repair of DNA damage induced by ACNU or TMZ. PLoS One 6: e19659, 2011.

14. Fu X, Tao L, Wang PY, Cripe TP and Zhang X: Comparison of infectivity and spread between HSV-1 and HSV-2 based oncolytic viruses on tumor cells with different receptor expression profiles. Oncotarget 9: 21348-21358, 2018.

15. Baer A and Kehn-Hall K: Viral concentration determination through plaque assays: Using traditional and novel overlay systems. J Vis Exp: e52065, 2014.

16. Han C and Yang C: Viral plaque analysis on a wide field-of-view, time-lapse, on-chip imaging platform. Analyst 139: 3727-3734, 2014

17. Chou TC: The median-effect principle and the combination index for quantitation of synergism and antagonism. In: Chou TC and Rideout DC (eds). Synergism and antagonism in chemotherapy. Academic Press, San Diego, pp61-102, 1991.

18. Khafif A, Schantz SP, Chou TC, Edelstein D and Sacks PG: Quantitation of chemopreventive synergism between (-)-epigallocatechin-3-gallate and curcumin in normal, premalignant and malignant human oral epithelial cells. Carcinogenesis 19: 419-424, 1998.

19. Livak KJ and Schmittgen TD: Analysis of relative gene expression data using real-time quantitative PCR and the 2(-Delta Delta C(T)) method. Methods 25: 402-408, 2001.

20. Vallin J and Grantham J: The role of the molecular chaperone $\mathrm{CCT}$ in protein folding and mediation of cytoskeleton-associated processes: Implications for cancer cell biology. Cell Stress Chaperones 24: 17-27, 2019.

21. Morata G and Ballesteros-Arias L: Cell competition, apoptosis and tumour development. Int J Dev Biol 59: 79-86, 2015.

22. Roos WP and Kaina B: DNA damage-induced cell death by apoptosis. Trends Mol Med 12: 440-450, 2006.

23. Mah LJ, El-Osta A and Karagiannis TC: gammaH2AX: A sensitive molecular marker of DNA damage and repair. Leukemia 24: 679-686, 2010.

24. Thomas SE, Malzer E, Ordóñez A, Dalton LE, van 't Wout EF, Liniker E, Crowther DC, Lomas DA and Marciniak SJ: p53 and translation attenuation regulate distinct cell cycle checkpoints during endoplasmic reticulum (ER) stress. J Biol Chem 288: 7606-7617, 2013.

25. Watanabe D: Medical application of herpes simplex virus. J Dermatol Sci 57: 75-82, 2010.

26. Aghi M, Rabkin S and Martuza RL: Effect of chemotherapy-induced DNA repair on oncolytic herpes simplex viral replication. J Natl Cancer Inst 98: 38-50, 2006.

27. Jahan N, Lee JM, Shah K and Wakimoto H: Therapeutic targeting of chemoresistant and recurrent glioblastoma stem cells with a proapoptotic variant of oncolytic herpes simplex virus. Int J Cancer 141: 1671-1681, 2017.

28. Norbury CJ and Zhivotovsky B: DNA damage-induced apoptosis. Oncogene 23: 2797-2808, 2004.

29. Borges HL, Linden R and Wang JY: DNA damage-induced cell death: Lessons from the central nervous system. Cell Res 18: 17-26, 2008. 
30. Prestwich RJ, Errington F, Diaz RM, Pandha HS, Harrington KJ, Melcher AA and Vile RG: The case of oncolytic viruses versus the immune system: Waiting on the judgment of Solomon. Hum Gene Ther 20: 1119-1132, 2009.

31. Holliday DL and Speirs V: Choosing the right cell line for breast cancer research. Breast Cancer Res 13: 215, 2011.

32. Melisko ME, Assefa M, Hwang J, DeLuca A, Park JW and Rugo HS: Phase II study of irinotecan and temozolomide in breast cancer patients with progressing central nervous system disease. Breast Cancer Res Treat 177: 401-408, 2019.

33. Bobustuc GC, Kassam AB, Rovin RA, Jeudy S, Smith JS, Isley B, Singh M, Paranjpe A, Srivenugopal KS and Konduri SD: MGMT inhibition in ER positive breast cancer leads to CDC2, TOP2A, AURKB, CDC20, KIF20A, Cyclin A2, Cyclin B2, Cyclin D1, $\mathrm{ER} \alpha$ and Survivin inhibition and enhances response to temozolomide. Oncotarget 9: 29727-29742, 2018.
34. Garza-Morales R, Gonzalez-Ramos R, Chiba A, Montes de Oca-Luna R, McNally LR, McMasters KM and Gomez-Gutierrez JG: Temozolomide enhances triple-negative breast cancer virotherapy in vitro. Cancers (Basel) 10: 144, 2018.

(i) (2) This work is licensed under a Creative Commons Attribution-NonCommercial-NoDerivatives 4.0 International (CC BY-NC-ND 4.0) License. 